\title{
Effects of alternation in some quasi-one-dimensional magnetic materials
}

Martin L. Kirk and William E. Hatfield

Department of Chemistry, The University of North Carolina, Chapel Hill, North Carolina 27599-3290

Myoung Soo Lah, Dimitri Kessissoglou, and Vincent L. Pecoraro

Department of Chemistry, University of Michigan, Ann Arbor, Michigan 48109-1055

Larry W. Morgan and John D. Petersen

Department of Chemistry, Clemson, University, Clemson, South Carolina 29634

Exchange coupling in $\mathrm{Cu}$ (II) and $\mathrm{Mn}$ (III) compounds with unusual structures is discussed. $\left\{\left[\mathrm{Cu} \text { (bipyrimidine) }(\mathrm{OH})\left(\mathrm{H}_{2} \mathrm{O}\right)\right]\left(\mathrm{ClO}_{4}\right)\right\}_{n}$ has an alternatingly bridged structure with alternating ferromagnetic $\left(+167.6 \mathrm{~cm}^{-1}\right.$ through the hydroxo bridge) and antiferromagnetic $\left(-79.8 \mathrm{~cm}^{-1}\right.$ through the bipyrimidine bridge) interactions. Copper(II) phthalate monohydrate has alternating next-nearest-neighbor exchange with $J=-12.3$ $\mathrm{cm}^{-1}$ and $\alpha=0.06$. This is the first member of this class. The compound $\mathrm{K}_{2}[\mathrm{Mn}$ (III) (salicylate) $\left.{ }_{2}\right]\left[\mathrm{Mn}\right.$ (III) (salicylate) $\left.{ }_{2}\right]\left[\mathrm{CH}_{3} \mathrm{OH}\right]_{2}$ has manganese ions in two environments alternating along the chain. A modified model for the chain is presented, and exchange coupling is found to be small since magnetic orbitals are not linked by the bridging ligand.

\section{INTRODUCTION}

The static antiferromagnetic alternating chain has received considerable theoretical and experimental attention in recent years because of the similarity of the chain to one-dimensional materials that undergo a spin-Peierls transition. ${ }^{1}$ Alternating chains with both superexchange interactions being antiferromagnetic have received the most attention, although only a small number of such compounds have been, magnetically and structurally characterized. ${ }^{2}$ Magnetic susceptibility data for these compounds have been analyzed by using closed-form expressions generated from theoretical results of calculations on rings of 12-14 spins that provide good approximations to the infinite chain limit. Little work has been done on alternating chains with spin $S>\frac{1}{2}$, on alternating chains with ferromagnetic and antiferromagnetic interactions, or on alternating chains with novel structures such as the alternating nextnearest-neighbor chain. Preliminary data on alternating chains with these features are presented here.

\section{THEORY}

The Heisenberg Hamiltonian for a one-dimensional system displaying alternating chain magnetism may be written as

$$
H=-2 J \sum_{i=1}^{N-2} S_{i} \cdot S_{i+2}-2 J \alpha \sum_{i=1}^{N / 2-1} S_{2 i} \cdot S_{2 i+1},
$$

where $J$ is the exchange constant associated with a particular exchange-coupled pair, and $\alpha J$ is the exchange constant associated with the adjacent unit. The ground state of an antiferromagnetic alternating chain with $+\alpha<1$ and ions having the same spin is a spin singlet. In this case the ground state is separated from the band of paramagnetic states by an energy gap $\Delta E$. It is not possible, at this time, to make general statements concerning the magnetic properties of more complicated alternating chains.

\section{FERROMAGNETIC AND ANTIFERROMAGNETIC EXCHANGE IN THE ALTERNATING CHAIN COMPOUND $\left[\mathrm{Cu}(\mathrm{bpm})(\mathrm{OH})\left(\mathrm{ClO}_{4}\right) \cdot \mathrm{H}_{2} \mathrm{O}\right]_{n}$}

The structure of the alternating chain $[\mathrm{Cu}(\mathrm{bpm})(\mathrm{OH})$ $\left.\left(\mathrm{ClO}_{4}\right) \cdot \mathrm{H}_{2} \mathrm{O}\right]_{N}$ (bpm $=2,2^{\prime}$-bipyrimidine $)$ is shown in Fig. 1. The most striking feature of this compound is the alternating hydroxo-bipyrimidine bridging arrangement in the plane of the copper ions.

The geometry about the individual copper ions is six coordinate. In the tetragonal plane about copper, there are two hydroxo oxygen atoms and two nitrogen atoms from the bipyrimidine. The axial sites are occupied by aquo ligans. The bipyrimidine ligands are planar, and the copper ion, the bridging hydroxo ligands, and the bipyrimidine ligand lie approximately in the same plane. A metrical parameter of particular interest is the $\mathrm{Cu}-\mathrm{O}-\mathrm{Cu}$ angle in the hydroxo-bridged fragment, which is $95.5^{\circ}$.

The magnetic susceptibility, which was determined with a Faraday balance in the range $77-300 \mathrm{~K}$ with an applied field of $7.5 \mathrm{kOe}$, displays a rounded maximum at $113 \mathrm{~K}$. The magnetic susceptibility data were analyzed with a 12-spin ring approximation. This model resulted in an excellent fit to the data with the best-fit magnetic parameters being $J_{1}=+167.6 \mathrm{~cm}^{-1}, J_{2}=-79.8 \mathrm{~cm}^{-1}$, and $g=2.25$.

Bipyrimidine is well known to facilitate antiferromagnetic interactions between transition-metal ions. ${ }^{3}$ The exchange coupling constants typically range from $-77.8 \mathrm{~cm}$ to $-118 \mathrm{~cm}^{-1}$. In order to ascertain the orbital nature of this superexchange pathway, Gaussian 88 ab initio calculations were performed on a pyrimidine subunit. The resultant highest occupied molecular orbitals show good overlap between copper $d_{x 2-y_{2}}$ and $p \sigma$ orbitals of the bridging ligand. These results permit an assignment of the exchange coupling constant of $-79.8 \mathrm{~cm}^{-1}$ to the bipyrimidine-bridge fragment of the alternating chain.

The effects of bridge geometry on the sign and magnitude of exchange coupling constants in di- $\mu$-hydroxobridged dimers is well known. ${ }^{4,5}$ There is a linear depen- 


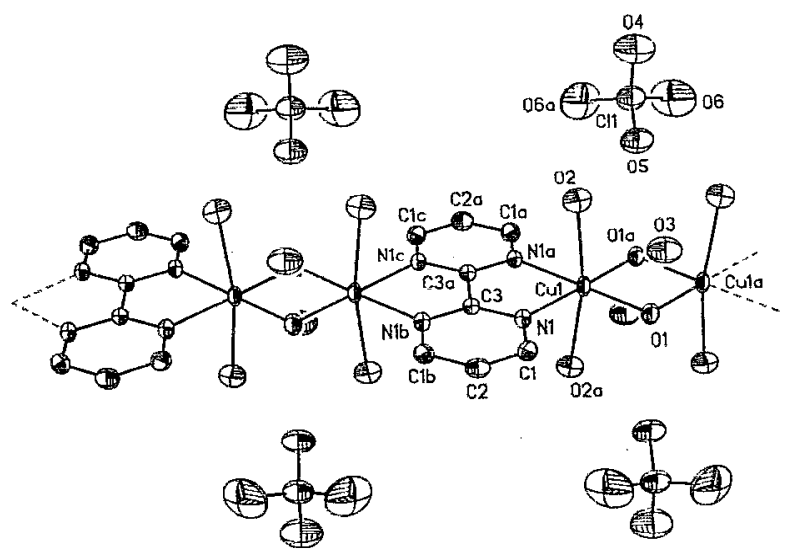

FIG. 1. The structure of $\left\{\mathrm{Cu} \text { (bipyrimidine) }(\mathrm{OH})\left(\mathrm{H}_{2} \mathrm{O}\right) \mathrm{CClO}_{4}\right\}_{n}$.

dence of the singlet-triplet splitting in five-coordinate dimers on the angle $\phi$ at the hydroxo bridge given by $2 J$ $\left(\mathrm{cm}^{-1}\right)=-74.53(\phi)+7270$. The singlet-triplet splittings for six-coordinate hydroxo-bridged copper (II) dimers deviate strongly toward more positive values from those predicted from the above cquation. Clcarly the exchange coupling constant of $+167.6 \mathrm{~cm}^{-1}$ corresponds to the exchange coupling in the di- $\mu$-hydroxo fragment of the alternating chain. $\left[\mathrm{Cu}(\mathrm{bpm})(\mathrm{OH})\left(\mathrm{ClO}_{4}\right) \cdot \mathrm{H}_{2} \mathrm{O}\right]$ represents only the third example of alternating ferromagnetic and antiferromagnetic exchange coupling in a onedimensional chain. The other two examples are $\mathrm{CuCl}_{3} \cdot(4-$ Bzpip) (Ref. 6) and $\mathrm{Cu}(\mathrm{hfac}) \cdot \mathrm{TEMPOL}^{7}$, which have $(J, \alpha)$ values estimated to be $\left(21 \mathrm{~cm}^{-1},-0.1\right)$ and $(12.9$ $\left.\mathrm{cm}^{-1},-0.004\right)$, respectively. The present analysis represents the first description of the magnetic properties of an alternating chain with negative $\alpha$ using the real spin basis functions.

\section{THE ALTERNATING NEXT-NEAREST-NEIGHBOR CHAIN}

The magnetic array and independent exchange coupling constants of the alternating next-nearest-neighbor chain represents one of the least understood linear chain systems. The compound that exhibits alternating nextnearest-neighbor interactions is copper(II) phthalate monohydrate. ${ }^{8}$

The Heisenberg Hamiltonian for a linear chain exhibiting nearest-neighbor and next-nearest neighbor exchange interactions is

$$
\begin{aligned}
H= & -2 J \sum_{i=1}^{N-1} S_{i} \cdot S_{i+1}-2 J \alpha \sum_{i=1}^{N / 2-1} S_{2 i-1} \cdot S_{2 i+1} \\
& -2 J \beta \sum_{i=1}^{N / 2-1} S_{2 i} \cdot S_{2 i+2}
\end{aligned}
$$

where $J$ is the exchange integral between a given site and its nearest neighbor, $\alpha$ is the ratio of the next-nearest neighbor to nearest-neighbor exchange on odd sites, and $\beta$ is the corresponding exchange parameter for even sites.

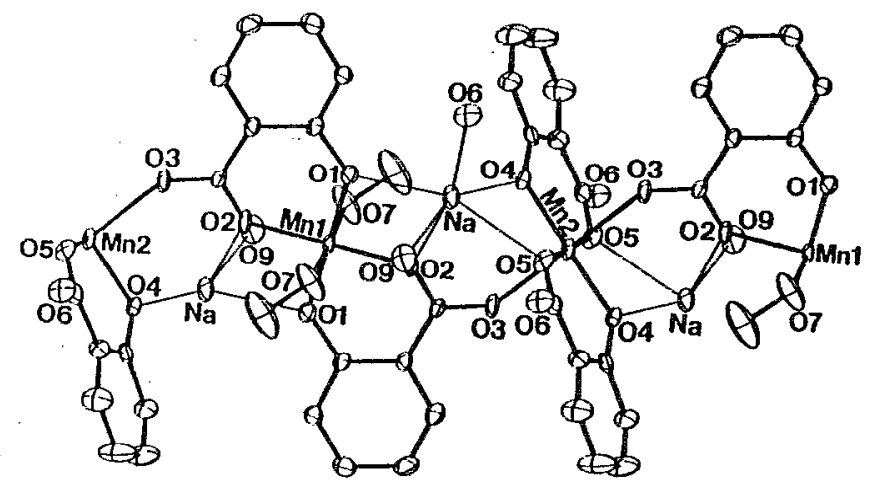

FIG. 2. The structure of $\left.\left.\mathrm{K}_{2}[\mathrm{Mn} \text { (III) (salicylate) })_{2}\right][\mathrm{Mn} \text { (III) (saliclate) })_{2}\right]$ $\left.\left[\mathrm{CH}_{3} \mathrm{OH}\right)_{2}\right]$.

This Hamiltonian, with $\beta$ set to zero since there is no exchange pathway connecting even sites, was used in the best-fit calculations of the theoretical results for 12 spins to the magnetic susceptibility data. These magnetic susceptibility data were collected in the range $1.8-100 \mathrm{~K}$ by using a PAR vibrating sample magnetometer. ${ }^{9}$ The best-fit parameters are $J=-12.3 \mathrm{~cm}^{-1}, \alpha=0.06$, and $g=2.19$. the $g$ valuc is in excellent agreement with the $g$ value determined from electron parametric resonance (EPR), and $\alpha$ is in the range estimated from an earlier ${ }^{2}$ approximate analysis. Since there are two different copper(II) ions in the structure, one may have expected a divergence of the magnetic susceptibility at low temperatures as a result of incomplete compensation of the spin. However, the similar $4+2$ coordination environment about the copper ions and the propensity for copper to display nearly isotropic $g$ values precludes ferromagnetic behavior. The confirmation of nearest-neighbor as well as alternating next-nearestneighbor exchange in $\mathrm{Cu}\left(\mathrm{H}_{2} \mathrm{O}\right)$ (pht) defines a new magnetic system with completely different thermodynamic properties from other linear chain compounds. This is the first compound in this class.

\section{ALTERNATING CHAINS WITH $S>\frac{1}{2}$}

Linear chain compounds of manganese(III) are rare. Single ions display complicated behavior due to a large number of excited electronic states and zero-field splittings. Magnetic susceptibility has been used to provide the bulk of information concerning these systems. As a further complication, the presence of a large single-ion anisotropy can induce anisotropy in the exchange interaction. The theory for anisotropic exchange in chains with $S>\frac{1}{2}$ is not well developed. Therefore, the exchange parameters absorb the effects of this anisotropy. In this section, the structure and magnetism of a manganese(III) chain compound that has manganese(III) ions in different sites which alternate along the chain will be discussed.

The structure of $\mathrm{K}_{2}\left[\mathrm{Mn}\right.$ (III) (salicylate) $\left.{ }_{2}\right][\mathrm{Mn}$ (III) (salicylate) $\left.\left.{ }_{2}\right]\left[\mathrm{CH}_{3} \mathrm{OH}\right)_{2}\right]$ is shown in Fig. 2. Two manganese(III) ions make up the magnetic unit of an $A-B$ infinite chain wherein the Jahn-Teller axes of the individual ions are canted by $82.9^{\circ}$. 
The $\mathrm{Mn}$ (III) ion $\mathrm{A}$ [Mn(1) in Fig. 2] is six coordinate, being bound by two bidentate salicylate ligands which form the tetragonal plane and axially by two methanol solvent molecules. This results in an elongated octahedral coordination environment about $\mathrm{Mn}$ (A) in which none of the $\mathrm{O}-\mathrm{Mn}(\mathrm{A})-\mathrm{O}$ angles differ more than $5^{\circ}$ from perfect $D_{4 h}$ values.

The $\mathrm{Mn}$ (III) ion B is also six coordinate, bound by two bidentate salicylate ligands forming the tetragonal plane and axially by two carboxylate oxygens from the Mn(III) A unit salicylates. The coordination environment about site $\mathbf{B}$ is more highly distorted than that about site $\mathbf{A}$, as evidenced by the fact that the $\mathrm{O}-\mathrm{M}(\mathrm{B})-\mathrm{O}$ bond angles deviate by as much as $6.7^{\circ}$ from the ideal $D_{4 h}$ angles.

The magnetic properties of $d^{4}$ high-spin Mn(III) ions in a magnetic field is quite complex, being complicated by exchange interactions, low-symmetry ligand fields, $g$-tensor anisotropy, and the Jahn-Teller effect. The following Hamiltonian applies to a linear array of isotropically exchange coupled $\mathrm{Mn}$ (III) ions:

$$
\begin{aligned}
H= & g_{\|} \beta \sum_{i=1}^{N}\left(H_{z} \cdot S_{i z}\right)+g_{\perp} \beta \sum_{i=1}^{N}\left(H_{x} \cdot S_{i x}+H_{y}: S_{i y}\right) \\
& -2 J \sum_{i=1}^{N}\left(S_{i} \cdot S_{i+1}\right]+D \sum_{i=1}^{N}\left[S_{i z}^{2}-\frac{1}{3} S(S+1)\right] \\
& +E \sum_{i=1}^{N}\left(S_{i y}^{2}+S_{i x}^{2}\right)
\end{aligned}
$$

Since the two sites are in different environments, there should be a separate $D, E, g_{\|}$, and $g_{\perp}$ for each ion. Thus, some approximations must be made while being careful not to compromise the ability to understand the properties of the system. Here, rhombic splitting will not be taken into account, and it will be assumed that the $g$ factors and zero-field splitting terms will be equal for both ions.

The magnetic moment of $\mathrm{K}_{2}\left[\mathrm{Mn}\right.$ (III) (salicylate) $\left.{ }_{2}\right]$ [Mn(III) (salicylate) $\left.\left.{ }_{2}\right]\left[\mathrm{CH}_{3} \mathrm{OH}\right)_{2}\right]$ falls from $4.8 \mu_{B}$ at 80 $\mathrm{K}$ in an applied magnetic field of $10 \mathrm{kOe}$ to $2.4 \mu_{B}$ at $1.9 \mathrm{~K}$. Similar behavior was observed in a 5 -kOe data set. A model similar to one used by Marathe and Mitra ${ }^{10}$ was employed. In their model the $N g^{2} \beta^{2} S(S+1) / 3 k T$ terms in the expression derived by Fisher ${ }^{11}$. for the isotropic Heisenberg nearest-neighbor chain was replaced by $\chi_{\text {zfs }}$, which was calculated by using the Zeeman terms, and the term in $D$ in the Hamiltonian given above. The final expression becomes

$$
\chi_{\text {chain }}=\chi_{\mathrm{zfs}} \frac{A+B x^{2}}{1+C x+D x^{3}},
$$

where the ratio of polynomials ${ }^{12}$ replaces the $[(1+U) /$ $(1-U)]$ term used by Mitra. The coefficients $A-D$ were selected to reproduce the results on the nearest-neighbor chain, and $x=|J| / k T$. The best least-squares fit of the theory to the experimental data yield the parameters $g_{\|}=1.95, g_{\perp}=2.09, J=-0.02 \mathrm{~cm}^{-1}$, and $D=-15$ $\mathrm{cm}^{-1}$. The theory reproduces the experimental data nearly exactly over the entire temperature range.
The low value of the exchange coupling constant may be understood in terms of the relative orientations of the magnetic orbitals. The superexchange pathway through the carboxylate group 02-C7-03 connects the $d_{x 2-y 2}$ orbital on $\mathrm{Mn}(\mathrm{A})$ with the $d_{z 2}$ orbital on $\mathrm{Mn}(\mathrm{B})$. Since there is no unpaired electron density in the $\mathrm{Mn} d_{x 2-y 2}$ orbital, exchange effects are not expected. The small exchange coupling detected is a result of excited-state orbital mixing permitted by low-symmetry components of the crystal field.

\section{CONCLUDING REMARKS}

Closed-form expressions and finite-size ring calculations have been used to model the magnetic behavior of a series of manganese(III) and copper(II) compounds with unusual alternating chain structures. The magnetic parameters obtained have been discussed in terms of magnetostructural correlations and electronic structure effects, and excellent agreement has been obtained between phenomenology, theory, and experiment.

\section{ACKNOWLEDGMENTS}

This research was supported by the National Science Foundation through Grant No. CHE-88-07498. Calculations were carried out at the Cornell National Supercomputer Facility. We gratefully acknowledge the support and assistance provided by personnel at the supercomputing center.

${ }^{1} \mathrm{~J}$. W. Bray, L. V. Interrantc, I. S. Jacobs, and J. C. Bonncr, in Extended Linear Chain Compounds, edited by J. S. Miller (Plenum, New York, 1983), p. 353.

${ }^{2}$ W. E. Hatfield and L. W. ter Haar, in Bioinorganic and Inorganic Copper Chemistry, edited by K. D. Karlin and J. Zubiata (Adenine Press, Guilderland, New York, 1986), p. 173.

${ }^{3}$ M. Julve, G. De Munno, G. Bruno, and M. Verdaguer, Inorg. Chem. 27, 3160 (1988).

${ }^{4}$ W. E. Hatfield, Comments Inorg. Chem. 1, 108 (1981).

${ }^{5}$ D. J. Hodgson, Prog. Inorg. Chem. 19, 173 (1975).

${ }^{6}$ H. J. M. De Groot, L. J. de Jongh, R. D. Willett, and J. Reedijk, J. Appl. Phys. 53, 8038 (1982).

${ }^{7}$ C. Benelli, D. Gatteschi, D. W. Carnegie, and R. L. Carlin, J. Am. Chem. Soc. 107, 2560 (1985).

${ }^{8}$ C. K. Prout, J. R. Carruthers, and F. J. C. Rossotti, J. Chem. Soc. A, 3350 (1971).

${ }^{9}$ P. J. Corvan, W. E. Estes, R. R. Weller, and W. E. Hatfield, Inorg Chem. 19, 1297 (1980).

${ }^{10}$ V. R. Marathe and S. Mitra, J. Chem. Phys. 78, 1557 (1983).

${ }^{11}$ M. E. Fisher, Am. J. Phys. 32, 343 (1964).

${ }^{12} \mathrm{~J}$. W. Hall, Ph. D. thesis, University of North Carolina, Chapel Hill, North Carolina, 1977. 